\title{
ASO Video Abstract: Delayed Gastric Emptying After Multivisceral Resection for Retroperitoneal Sarcoma
}

\author{
Marco Baia, MD ${ }^{1}$, Lorenzo Conti, $\mathbf{M D}^{2}$, Sandro Pasquali, MD, $\mathbf{P h D}^{\mathbf{1}}$, Catherine Sarre-Lazcano, $\mathrm{MD}^{\mathbf{1}}$, \\ Carlo Abatini, $\mathrm{MD}^{1}$, Stefano Piero Bernardo Cioffi, $\mathrm{MD}^{1}$, Serena Della Valle, $\mathrm{MD}^{3}$, Giorgio Greco, $\mathrm{MD}^{4}$, \\ Raffaella Vigorito, $\mathrm{MD}^{4}$, Amanda Casirati, $\mathrm{RD}^{3}$, Paolo Proto, $\mathrm{MD}^{5}$, Cecilia Gavazzi, $\mathrm{MD}^{3}$, \\ Alessandro Gronchi, $\mathrm{MD}^{1}$, and Marco Fiore, MD, FACS ${ }^{1}$
}

${ }^{1}$ Sarcoma Service, Department of Surgery, IRCCS Fondazione Istituto Nazionale dei Tumori di Milano, Milan, Italy; ${ }^{2}$ Ospedale Galmarini di Tradate, ASST Settelaghi, Tradate, Varese, Italy; ${ }^{3}$ Clinical Nutritional Service, IRCCS Fondazione Istituto Nazionale dei Tumori di Milano, Milan, Italy; ${ }^{4}$ Department of Radiology, IRCCS Fondazione Istituto Nazionale dei Tumori di Milano, Milan, Italy; ${ }^{5}$ Anesthesiology Department, IRCCS Fondazione Istituto Nazionale dei Tumori di Milano, Milan, Italy

Data from 100 consecutive patients treated for primary retroperitoneal sarcoma (RPS) were analyzed from our institutional prospectively maintained database from January 2019 to April 2020 (https://doi.org/10.1245/s10434021-11154-z). Following multivisceral resection, delayed gastric emptying (DGE) is a clinically relevant event that can be caused by an underlying complication. Prompt diagnosis and treatment of both DGE and any underlying complications lead to full recovery in all cases.
Supplementary Information The online version contains supplementary material available at https://doi.org/10.1245/s10434021-11261-x.

FUNDING No funding has been used for this publication.

Publisher's Note Springer Nature remains neutral with regard to jurisdictional claims in published maps and institutional affiliations.

(C) Society of Surgical Oncology 2021

M. Fiore, MD, FACS

e-mail: marco.fiore@istitutotumori.mi.it 